\title{
Molecular diversity and natural products
}

\author{
Giovanni Appendino • Luca Banfi
}

Published online: 21 April 2011

(C) Springer Science+Business Media B.V. 2011

Natural products have long represented the mayor source of drugs, and even today they remain at the forefront of pharmaceutical research. Between 1982 and 2002, 28\% of the New Chemical Entities (NCE) approved by the FDA were natural products or derivatives of natural products [1], a very high share, since only less than $1 \%$ of the characterized organic compounds are of natural origin. Natural products are still playing a major role in the realm of anti-cancer and antiinfective drug discovery, but their relevance in other fields has constantly declined in the wake of the introduction of "hungry" methodologies like combinatorial chemistry and ultrahigh throughput screening. More recently, a resurgence is being witnessed, owing to the growing awareness that natural products represent not only potential New Chemical Entities (NCE), but also privileged structures to explore the chemical space around a target. Plants, fungi, bacteria, and marine organisms make medicinally interesting compound for reasons of their own, that not necessarily overlap with the needs. Nevertheless, natural products are made by enzymes, and their shape must be complementary to that of a biological surface. This makes them privileged structures in terms of chemical space of biological relevance. Natural products are "sticky" toward biological surfaces not only for the presence of functional groups capable to bind biomolecules through the whole arsenal of non-covalent interaction (hydrogen- and

\footnotetext{
G. Appendino $(\bowtie)$

Department of Chemical, Food, Pharmaceutical and Pharmacological Sciences, University of Eastern Piedmont, via Bovio 6, 28100 Novara, Italy

e-mail: appendino@pharm.unipmn.it

L. Banfi $(\bowtie)$

Department of Chemistry and Industrial Chemistry, University

of Genova, via Dodecaneso, 31-16146 Genova, Italy

e-mail: banfi@chimica.unige.it
}

halogen-bonding, $\pi$-stacking, dipolar interactions), but also for their embedding in a molecular framework that can fits, in terms of van der Waals interactions, a biological surface.

Although only about 10,000 natural products are discovered every year, their high structural diversity guarantees coverage of the virtual chemical space larger than any collection of synthetic compounds. Indeed, certain functional groups or combinations of functional groups such as $\beta$-lactams and enediynes owe their popularity in organic chemistry to their occurrence in natural products. Natural compounds have not only a higher chance to show biological activity and become "hits," but also a higher chance to survive the drug development pipeline [2]. Thus, they have better chances to become drugs, despite an often substantial deviation from the classical rules for drug-likeness. Natural products typically have more stereogenic centers and more architectural complexity than synthetic molecules designed by medicinal chemists. They have more carbon, hydrogen, and oxygen atoms and less nitrogen atoms.

Another reason for the higher chance of success of natural products compared to randomly synthesized molecules could be the optimization of their function carried out by evolution. Nature is not only a supreme synthetic chemist, but also a supreme pharmacist. The high diversity of natural substances can be further increased by semisynthesis of libraries of natural product derivatives, aided by the dramatic improvements in combinatorial synthesis and in synthesis automation achieved in the past two decades. This approach is, however, limited to natural products that can only be obtained by extraction or fermentation in "synthetically useful" amounts. Furthermore, not all parts of a natural product can be modified, precluding the exploration of a significant part of their pharmacophoric space. Therefore, another emerging field is the total synthesis of "natural-like" fully synthetic compounds $[3,4]$. This strategy takes advantage 
of the "biology-validated" structure of natural products, yet overcoming the synthetic issues related to the synthesis of analogs. Finally, computational methods can also be precious in boosting the application of natural products, through virtual screening of natural products databases.

This special issue of Molecular Diversity was conceived in order to exemplify all these aspects, collecting a series of invited papers from researchers chosen among the main players in this field. The quality of the received manuscripts was quite high, and provides a comprehensive overlook on the connections between natural products and chemical diversity, as seen from different perspectives.

An article, written by Jose L. Medina-Franco et al., is dedicated to the virtual screening of the natural product chemical space. The authors have carried out a virtual screen of a large database of natural products using a validated homology model of the catalytic domain of DNMT1 (DNA methyltransferase). This is the first in silico study of a large database of natural products toward the identification of novel compounds with inhibitory activity of DNMTs. 89,000 compounds were evaluated and the study led to the selection of 56 hits. Interestingly, one of them was already experimentally reported to be an actual inhibitor, thus validating the computational approach.

Three other articles describe the experimental discovery of interesting biological activities in a series of natural products. The paper by Matthias Hamburger et al. focuses on the discovery of new GABAA receptor modulators from a medicinal plant. HPLC profiling enabled the isolation and characterization of four flavonoids capable of affecting the activity of this ion channel. The study demonstrates that it is still possible to individuate new pharmaceutical leads by a careful study of the traditional medicine remedies. Nunziatina De Tommasi et al. have reviewed the discovery of small-molecule HAT (Histone Acetyl Transferases) modulators from naturally occurring substances of their derivatives. These modulators are likely to find application in the anti-cancer drugs field. Finally, Claudiu T. Supuran reported the discovery of new natural inhibitors of carbonic anhydrase (CA). Inhibition of human $\alpha$-CAs with sulfonamides has been exploited for decades for various pharmacological applications (e.g., diuretics, anti-glaucoma drugs). This paper discloses new chemotypes based on natural substances and explains the mechanism of inhibition.

Two additional papers present the semisynthesis of analogs of natural substances endowed with important biological and pharmacological activity in the field of anti-cancer drugs. Karl Heinz Altmann et al. have reviewed the semisynthesis of various epothilone analogs. This is a perfect example of the exploration of diversity through semisynthesis, starting from complex natural molecules endowed with a very important biological activity as microtubule stabilizers. Donald
Poirier et al. have also prepared a library based on natural products: A collection of steroid derivatives with two levels of molecular diversity was prepared to optimize the antiproliferative activity on leukemia HL-60 cells.

The remaining three papers are related to the synthesis of natural product like compounds, developing new synthetic methodologies and strategies toward this goal.

Marco Anderluh and Anna Bernardi et al. have reported the synthesis of a brilliantly designed focused library of glycomimetics as DC-SIGN (Dendritic cell-specific intercellular adhesion molecule 3-grabbing nonintegrin) antagonists. The field of oligosaccharides and glycomimetics represents a typical case where semisynthesis from natural substances is not easily affordable. Thus, they anchored a rationally designed protein recognition domain to a natural carbohydrate scaffold (mannose). The synthesized compounds were tested using a DC adhesion assay to mannan-coated plates and the results demonstrated that the majority of the new derivatives inhibit DC adhesion more potently, by up to 2 orders of magnitude, than the starting lead. In addition, docking studies allowed qualitative rationalization of the results. Also, with the aim to aid glycomimetic synthesis, Francesco Nicotra et al. optimized the direct glycosylation of monosaccharides using Fischer glycosylation under ultrasound activation. This methodology allows the direct, diversity-oriented, synthesis of unprotected glycosides possessing suitable functional groups that can be further manipulated by chemoselective reactions. Finally, Ali Khalafi-Nezhad reported an improved methodology for the combinatorial synthesis of polyhydroxyl aromatic compounds related to arzanol and other biologically active natural compounds. The described methodology makes use of silica-sulfuric acid in an improved procedure for the coupling of two phenolic building blocks.

In conclusion, we think that this special issue can give a general idea of the current state of the art in the virtual and synthetic exploration of the chemical space occupied by natural substances, their semisynthetic derivatives and of natural product like molecules, and will, hopefully, stimulate and promote research in this germane area of research.

\section{References}

1. Clardy J, Walsh C (2004) Lessons from natural molecules. Nature 432:829-837. doi:10.1038/nature03194

2. Kong D-X, Ren W, Lu W, Zhang H-Y (2009) Do biologically relevant compounds have more chance to be drugs? J Chem Inf Model 49:2376-2381. doi:10.1021/ci900229c

3. Davies HML (2009) Synthetic lessons from nature. Nature 459:786-787. doi:10.1038/459786a

4. Nandy JP, Prakesch M, Khadem S, Reddy PT, Sharma U et al (2009) Advances in solution- and solid-phase synthesis toward the generation of natural product-like libraries. Chem Rev 109: 1999-2060. doi:10.1021/cr800188v 